\title{
Synthesis, Characterization, and Application of Activated Carbon/CuBTC Composite for Improving Separation Performance of CO2/H2 Binary Mixture
}

Kourosh Esfandiari ( $\nabla$ kourosh.esfandiari@gmail.com )

Babol Noshirvani University of Technology https://orcid.org/0000-0002-0957-3361

Ali Asghar Ghoreyshi

Babol Noshirvani University of Technology

Original Research Full Papers

Keywords: MOF, CuBTC, composite material, gas separation, CO2 sequestration

Posted Date: February 2nd, 2021

DOI: https://doi.org/10.21203/rs.3.rs-175612/v1

License: (c) (1) This work is licensed under a Creative Commons Attribution 4.0 International License.

Read Full License 


\title{
Synthesis, Characterization, and Application of Activated Carbon/CuBTC Composite for Improving Separation Performance of $\mathrm{CO}_{2} / \mathrm{H}_{2}$ Binary Mixture
}

\author{
Kourosh Esfandiari*, Ali Asghar Ghoreyshi \\ Chemical Engineering Department, Babol Noshirvani University of Technology, \\ Shariati Street, Babol, Iran \\ Corresponding Author E-Mail: kourosh.esfandiari@gmail.com
}

ORCID: 0000-0002-0957-3361

\begin{abstract}
In this research, composites of a well-known metal-organic framework, CuBTC, and activated carbon (AC) are synthesized through solvothermal method and their performance is evaluated from viewpoint of $\mathrm{CO}_{2} / \mathrm{H}_{2}$ separation. For this purpose, different amounts of functionalized AC are incorporated in CuBTC structure and a volumetric-chromatographic technique is employed to measure the selectivity of $\mathrm{CO}_{2}$ over $\mathrm{H}_{2}$. XRD patterns, BET surface area, and FESEM images are employed to determine textural properties of as-synthesized samples. It is demonstrated that, while crystalline structure of $\mathrm{CuBTC}$ is not affected by incorporation of AC particles, adding proper amounts of functionalized $\mathrm{AC}$ to $\mathrm{CuBTC}$ matrix can cause higher $\mathrm{CO}_{2} / \mathrm{H}_{2}$ separation factor at low pressure and $298 \mathrm{~K}$. Obtained results suggest that adding $0.050 \mathrm{~g} \mathrm{AC}$ to CuBTC can increase the $\mathrm{CO}_{2} / \mathrm{H}_{2}$ selectivity up to around 32 which is double the separation factor of bare CuBTC (i.e. $\approx 15$ ). Enhanced hydrogen separation behavior might be attributed to the presence of carbonyl and nitrile functional groups exhibiting $\mathrm{CO}_{2}$ adsorption affinity on the surface of $\mathrm{AC}$ added to the optimum CuBTC structure.
\end{abstract}

Key words: $\mathrm{MOF}, \mathrm{CuBTC}$, composite material, gas separation, $\mathrm{CO}_{2}$ sequestration 


\section{Introduction}

Hydrogen energy is known as a clean and carbon-free energy source which produces almost zero pollution during combustion. The most commercialized process worldwide which produces around $95 \%$ of hydrogen is steam methane reforming (SMR) [1]. The final product of SMR contains mostly $\mathrm{H}_{2}$ and some impurities such as $\mathrm{CO}_{2}, \mathrm{CO}, \mathrm{N}_{2}$, and $\mathrm{H}_{2} \mathrm{O}$ in less extent. Consequently, these $\mathrm{H}_{2}$-dominated streams should be subjected to further purification to be used as a clean source of energy. Among these contaminants, $\mathrm{CO}_{2}$ has the most proportion $[2,3]$ and therefore $\mathrm{H}_{2}$ enrichment by means of $\mathrm{CO}_{2}$ sequestration has a particular importance.

Metal-organic frameworks (MOFs) are newly evolved class of porous solids with superior adsorption/separation characteristics. Tunable structure, high specific surface area (SSA), and high pore volume of MOFs make them an alternative candidate for numerous applications [4]. Nevertheless, it is demonstrated that the large void space within the MOF matrix does not completely participate in adsorption/separation processes [5]. An available approach to compensate the week interactions between the guest molecules and the internal pores can be the incorporation of second porous structures, especially carbonaceous materials, within the primary MOF matrix to form a composite material. Carbon nanotubes (CNTs) [6-9], graphene oxides (GrOs) [10-12], and activated carbons (ACs) are the main carbon-based materials which have been incorporated within MOF structures. With regard to $\mathrm{AC} / \mathrm{MOF}$ composites where $\mathrm{AC}$ is incorporated into MOF matrix, Bajaj et al. [13], incorporated AC particles within CuBTC matrix with the purpose of higher methane adsorption. Somayajulu Rallapalli et al. [14], assessed the potential of incorporating microporous AC in MIL-101 to achieve higher $\mathrm{H}_{2}$ uptakes. Lee and Park [15], enhanced the $\mathrm{H}_{2}$ storage capacity of a well-known Zn based MOF, i.e. MOF-5, by doping platinum on AC particles and incorporation of AC within MOF matrix. Prabhakaran and Deschamps [16] demonstrated the higher hydrogen adsorption of MIL-101 modified by AC and lithium doping. Mahmoodi et al. [17] 
removed Acid Green 25 (AG25) and Reactive Yellow 186 (RY186) dyes from colored wastewaters using AC incorporated MIL-101(Cr) and demonstrated that higher amounts of AC can enhance dye removal ability of the synthesized composite. McHugh et al. [18] prepared a composite material where STAM-17-OEt MOF incorporated into the commercially available BPL activated carbon (i.e. STAM-17-OEt@BPL) and showed enhance adsorptive characteristics of composite in comparison to both primary MOF and carbon materials. Recently, we showed that hydrogen capacity of CuBTC can be enhanced by incorporation of adequate amount of AC within the MOF matrix [19]. In this research, a CuBTC structure with enhanced textural characteristics was taken into account and different amounts of functionalized AC were incorporated within the MOF matrix to form a composite material. Finally, the performance of as-synthesized AC/CuBTC composites was evaluated from the viewpoint of $\mathrm{CO}_{2}$ sequestration from 50:50 (v:v) binary mixture of $\mathrm{CO}_{2} / \mathrm{H}_{2}$ using volumetric-chromatographic measurement.

\section{Materials and Methods}

\subsection{Chemicals}

Copper (II) nitrate trihydrate $\left(\mathrm{Cu}\left(\mathrm{NO}_{3}\right)_{2} .3 \mathrm{H}_{2} \mathrm{O}\right)$, benzene-1,3,5-tricarboxylic acid (trimesic acid or $\left.\mathrm{H}_{3} \mathrm{BTC}\right)$, nitric acid $\left(\mathrm{HNO}_{3}, 65 \%\right.$ wt.), and pure ethanol were purchased from Merck (Germany) and were used as received without further purification. The purity of $\mathrm{H}_{2}$ and $\mathrm{CO}_{2}$ were $99.999 \%$ and $99.95 \%$, respectively. Furthermore, $\sim 50: 50(\mathrm{v} / \mathrm{v})$ mixture of $\mathrm{H}_{2} / \mathrm{CO}_{2}$ was purchased from FaranSanat Mehr Co., Tehran, Iran. Acid functionalized activated carbon (AC) was provided from our previous research with proven carboxyl, carbonyl, and nitro functional groups on its surface and used without further operation [19]. It should be noted that, while carboxyl groups play the role of nucleation sites for AC/MOF composite formation, carbonyl and nitro groups have the merit of high $\mathrm{CO}_{2}$ affinity desirable for enhanced $\mathrm{CO}_{2} / \mathrm{H}_{2}$ separation purposes [20]. 


\subsection{Synthesis of Pristine CuBTC}

Synthesis of CuBTC begins by dissolving $1.75 \mathrm{~g}$ of Copper (II) nitrate trihydrate (metal salt) and $1.0 \mathrm{~g}$ of $\mathrm{H}_{3} \mathrm{BTC}$ (organic ligand) in $60 \mathrm{ml}$ pure ethanol. Afterward the mixture is stirred for 30 minutes before being transferred into a $100 \mathrm{ml}$ Teflon-lined stainless steel autoclave. The autoclave is capped tightly and heated up to $130{ }^{\circ} \mathrm{C}$ and kept in this temperature for $16 \mathrm{~h}$. After that, the autoclave is cooled down to RT and CuBTC crystals are separated by 3 times centrifugation (9000 rpm for $10 \mathrm{~min}$ ) and washed with ethanol each. Finally, the crystals are dried at $100^{\circ} \mathrm{C}$ overnight.

\subsection{Synthesis of AC/CuBTC Composite}

Synthesis of $\mathrm{AC} / \mathrm{CuBTC}$ composite is almost similar to what described for pure CuBTC synthesis. For this purpose, the desired amount of acid-treated AC is introduced into the preliminary MOF solution (i.e. the mixture of metal salt, ligand, and solvent) and the solution is then stirred (15 mins) and ultrasonicated $(15 \operatorname{mins}, 100 \mathrm{KW}, 400 \mathrm{~Hz})$ prior to being transferred into the autoclave. The rest of the procedure is entirely similar to the synthesis of pristine CuBTC. Table (1) summarizes the different levels of AC which are added into the CuBTC synthesis solution to form $A C / C u B T C$ composites. It is worth mentioned that, adding more than $0.050 \mathrm{~g}$ of AC into the synthesis solution resulted in formation of a dispersed black solid phase in the final product which was supposed to be un-reacted AC. Hence, the upper threshold for AC content in the synthesis solution was chosen to be $0.050 \mathrm{~g}$.

Table1 Summary of $A C / C u B T C$ composites and the amount of $\mathrm{AC}$ in their structure

\begin{tabular}{cc}
\hline Amount of $A C(\mathrm{~g})$ & AC/CuBTC Composite \\
\hline 0.003 & $A C-003 /$ CuBTC \\
0.005 & $A C-005 / C u B T C$ \\
0.010 & $A C-010 /$ CuBTC \\
0.050 & $A C-050 / C u B T C$ \\
\hline
\end{tabular}




\subsection{Characterization Techniques}

The powder X-ray diffraction (PXRD) patterns were collected on a PANalytical X'Pert Pro diffractometer (The Netherlands) in the range of $5^{\circ}$ to $45^{\circ}(2 \theta)$. In addition, Scherrer equation was employed to determine the crystal size of as-synthesized samples based on XRD data according to the following equation [21]:

$$
\tau=\frac{K \cdot \lambda}{\beta \cdot \cos \theta}
$$

where $\tau$ is the crystal size $(\mathrm{nm}), K$ is a dimensionless shape factor which is typically considered equal to $0.89, \lambda$ is the $\mathrm{X}$-ray wavelength $(0.154 \mathrm{~nm}$ in the case of $\mathrm{CuKa} 1), \beta$ is the full width at half maximum intensity of the peak (fwhm) in radians, and $\theta$ is the Bragg angle of the diffraction peak in radians. Brunauer-Emmett-Teller (BET) surface area of as-synthesized samples was determined by Belsorp Mini II (Japan). The morphology and structure of samples were studied with field emission scanning electron microscope (FESEM) images using Tescan Mira3 FEG (Czech Republic) with an acceleration voltage of $5.0 \mathrm{kV}$. Finally, the composition of gas mixtures was analyzed by Agilent 7890A gas chromatograph (USA) using helium as the sweeping gas. In addition, to determine $\mathrm{CO}_{2} / \mathrm{H}_{2}$ separation factor of samples, a volumetricchromatographic technique was used which is described in the following section in detail.

\subsection{Volumetric-Chromatographic Measurements}

Single gas adsorption measurements were carried out in an apparatus comprising of two similar vessels so-called "Load Cell" and "Adsorption Cell" (Figure 1) which are connected to each other with a valve (i.e. adsorption valve, Figure 1). The apparatus is equipped to a sensitive pressure sensor (Leo 2, Keller, Switzerland) to measure the pressure inside the load cell and whole volume of the apparatus. In addition, to keep the temperature constant during adsorption 
process, the cells are placed in a water bath (Memmert WaterBath WNB, Germany) capable to control the bath temperature using an electrical heating system. The adsorption pressure was increased gradually in order to draw the adsorption isotherm for each sample which was previously placed in "Adsorption Cell". It is worth noting that, the samples were activated by means of heating at $150^{\circ} \mathrm{C}$ overnight prior to each adsorption experiment.

For binary gas experiments the aforementioned apparatus was equipped to a precise needle valve (known as metering valve) to take a low pressure sample from the gaseous mixture maintained above the adsorbent surface after the equilibrium reached (Figure 1). The obtained sample was then injected into the gas chromatograph to determine the $\mathrm{CO}_{2} / \mathrm{H}_{2}$ composition.

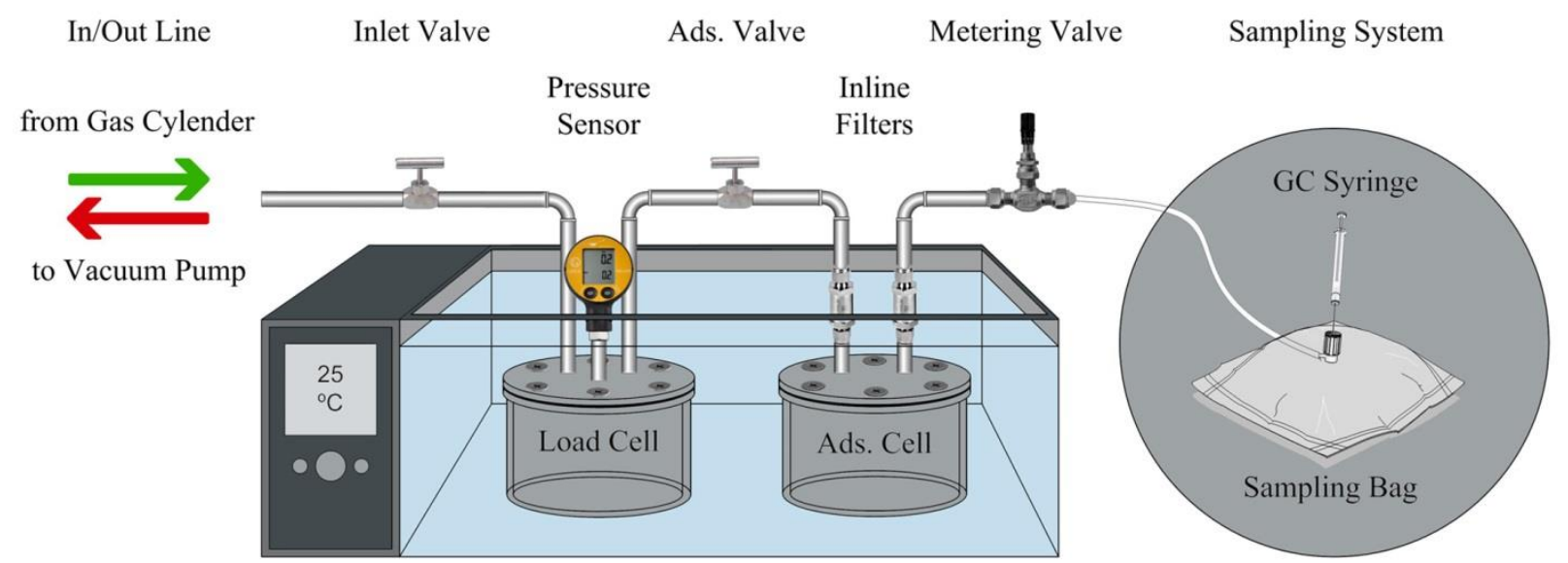

Fig1 Schematic diagram of volumetric adsorption apparatus equipped to a sampling line

Since the composition of the obtained samples from the adsorption cell (which is determined by gas chromatography) is not identical with the composition of the adsorbed phase within the adsorbent, an analytical approach was employed to determine the amounts of competitive adsorption of each $\mathrm{CO}_{2}$ and $\mathrm{H}_{2}$ on the adsorbent [21]. For this purpose, assume that a known amount of gas mixture available in the "Load Cell", is expanded into the "Adsorption Cell" and partially adsorbed on a certain mass of adsorbent, $\mathrm{m}^{s}$. The overall mass balance during this adsorption process can be written as Eq. (2), where $m^{*}(\mathrm{~kg})$ is the mass of initial gas stored in the 
Load Cell, $m^{f}(\mathrm{~kg})$ is the mass of gas remained on the surface of the solid (not adsorbed), and $m$ $(\mathrm{kg})$ is the mass adsorbed on the porous solid after the equilibrium reached. This material balance is also true for each component $i$ as well (Eq. 3). The purpose of this procedure is to determine the mass of component $i$ adsorbed on the porous solid $\left(m_{i}\right)$.

$$
m^{*}=m^{f}+m
$$

$m_{i}^{*}=m_{i}^{f}+m_{i}$

the total mass remained on the surface of the solid $\left(\mathrm{m}^{f}\right)$ can be found as below:

$m^{f}=\rho^{f}\left(V^{*}-V^{V}\right)$

where $\rho^{f}$ is the density of gas at the bulk phase $\left(\mathrm{kg} / \mathrm{m}^{3}\right)$ on the surface of the solid, $V^{*}$ is the total volume $\left(\mathrm{m}^{3}\right)$ of the experimental apparatus (Figure 1), and $V^{V}$ is the void volume $\left(\mathrm{m}^{3}\right)$, which indicates the inaccessible volume to adsorbing molecules in the Adsorption Cell. We have:

$V^{*}=V_{L C}+V_{A C}$

where $V_{L C}$ and $V_{A C}$ denote for the volume of Load Cell and Adsorption Cell, respectively. $V^{V}$ is considered to be equal to the volume occupied by the adsorbent $\left(V^{V}=V^{S}\right)$. Moreover, the density of gas phase can be determined based on the real gas equation of state:

$\rho^{f}=\frac{P M^{f}}{Z R T}$

where, $P, T$, and $R$ are pressure $(\mathrm{kPa})$, temperature $(\mathrm{K})$ and universal gas constant $\left(\mathrm{kPa} \cdot \mathrm{m}^{3} / \mathrm{mol} . \mathrm{K}\right)$, respectively. $Z$ is the compressibility factor of the sorptive gas mixture which is a function of pressure, temperature, and gas phase composition. $M^{f}(\mathrm{~kg} / \mathrm{kmol})$ is the molecular weight of gas mixture defined as:

$M^{f}=\sum_{i=1}^{N} M_{i} y_{i}$ 
where, $y_{i}$ is the gas molar composition and $M_{i}(\mathrm{~kg} / \mathrm{kmol})$, denotes the molecular weight of each constituent. Eq.(4) can be also written for each component $i$ in gas mixture remained on the surface of the solid at the equilibrium state:

$m^{f} \omega_{i}^{f}=\rho^{f} \omega_{i}^{f}\left(V^{*}-V^{V}\right)$

where, $\omega_{i}^{f}$ is the mass composition of component $i$ in the bulk gas phase (not adsorbed) which can be determined experimentally by gas chromatography. Equation (9) can be expressed in a reduced form as:

$m_{i}^{f}=\rho_{i}^{f}\left(V^{*}-V^{V}\right)$

by combining Eq. (9) with overall mass balance for component $i$ (Eq. 3), we have:

$m_{i}^{*}=\rho_{i}^{f}\left(V^{*}-V^{V}\right)+m_{i}$

rearranging Eq.(11) to obtain the mass of each component in the adsorbed phase, gives:

$m_{i}=\left(m_{i}^{*}-\rho_{i}^{f} V^{*}\right)+\rho_{i}^{f} V^{V}$

$m_{i}=\Omega_{i}+\rho_{i}^{f} V^{V}$

where $\Omega_{i}$ is the reduced mass adsorbed of component $i$. As an auxiliary quantity for $\Omega_{i}$, we have:

$\Omega_{i}=m_{i}^{*}-\rho_{i}^{f} V^{*}$

therefore, $m_{i}$ can be determined by means of Eq. (12) and taking Eq. (13). Finally, separation factor of component $\alpha$ (i.e. $\mathrm{CO}_{2}$ ) over component $\beta$ (i.e. $\mathrm{H}_{2}$ ) can be calculated using Eq. (14), where $x$ and $y$ are associated with the molar fractions of each component at the solid and gas phases, respectively. Moreover, superscripts $a$ and $f$ denote for adsorbed phase (determined by theoretical concept described above) and remained phase (not adsorbed) (determined by GC analysis), respectively.

$S_{\alpha \beta}=\frac{\left(x_{\alpha}^{a} / y_{\alpha}^{f}\right)}{\left(x_{\beta}^{a} / y_{\beta}^{f}\right)}$ 


\section{Results and Discussion}

\subsection{XRD Analysis}

Figure (2) shows the PXRD patterns of bare $C u B T C$ and $A C / C u B T C$ composite. Vividly, there is a good consistency between the position of XRD peaks associated with the CuBTC samples and what reported in the literature [22-24]. All samples possess sharp peaks at around 6.8, 9.6, 11.7, and 13.5 degrees which can be respectively assigned to (200), (220), (222), and (400) crystal planes of CuBTC. Moreover, the position of peaks associated with $A C / C u B T C$ composite does not shift in comparison to $C U B T C$ sample. This finding confirms that the crystalline matrix of CuBTC is not affected by AC incorporation.

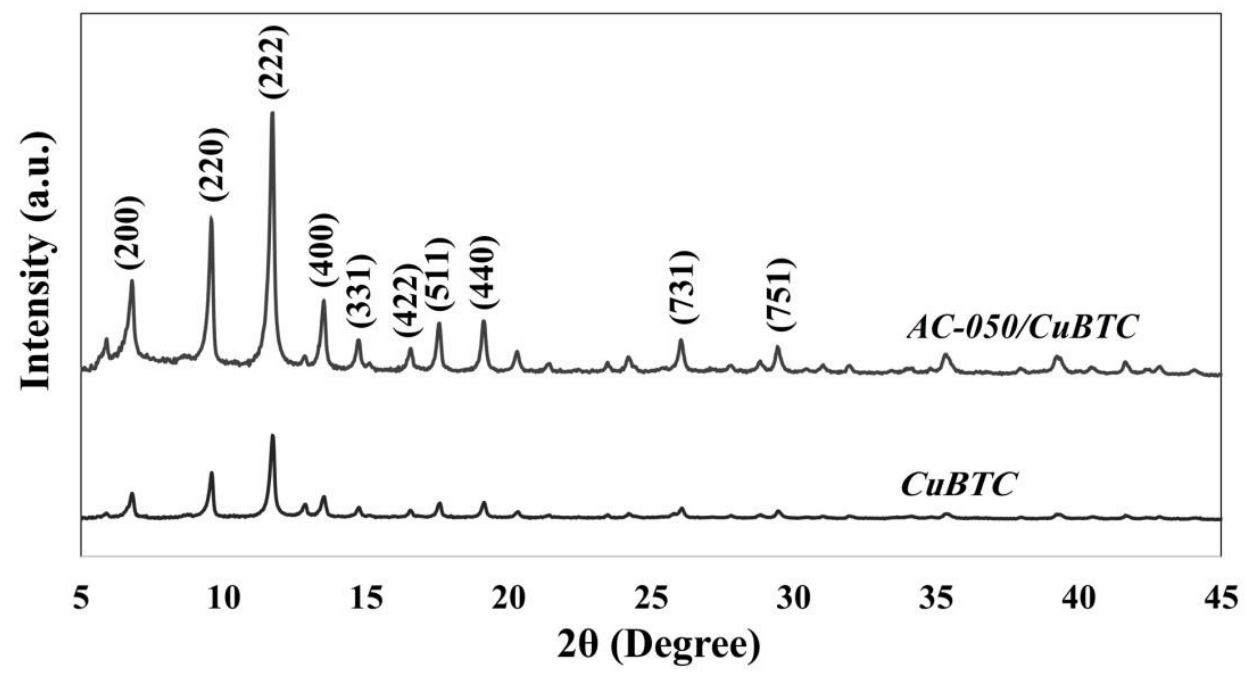

Fig2 PXRD patterns of bare $C u B T C$ and $A C-050 / C u B T C$ composite

In the case of crystal size by Scherrer equation, as is summarized in Table (2), With regard to composite sample, although the mean crystal size of $A C / C u B T C$ composite decreased in comparison to bare $\mathrm{CuBTC}$ probably due to amorphous nature of $\mathrm{AC}$ incorporated, the height of PXRD peaks increased. We believe that, this observation can be due to the formation of more ordered CuBTC structure in the presence of carboxyl functionalized AC which plays the role of nucleation sites. 
Table2 Mean crystal size of $C u B T C$ and $A C / C u B T C$ composite using Scherrer equation

\begin{tabular}{cccccc}
\hline \multicolumn{1}{c}{ CuBTC } & \multicolumn{3}{c}{ AC-050/CuBTC } \\
\hline $\begin{array}{c}\text { Peak Position } \\
(\mathbf{d e g}, \mathbf{2 \theta})\end{array}$ & $\begin{array}{c}\mathbf{f w h m} \\
(\mathbf{d e g})\end{array}$ & $\begin{array}{c}\boldsymbol{\tau} \\
(\mathbf{n m})\end{array}$ & $\begin{array}{c}\text { Peak Position } \\
\mathbf{( d e g}, \mathbf{2 \theta})\end{array}$ & $\begin{array}{c}\mathbf{f w h m} \\
\mathbf{( d e g})\end{array}$ & $\begin{array}{c}\boldsymbol{\tau} \\
(\mathbf{n m})\end{array}$ \\
\hline 6.859 & 0.200 & 39.335 & 6.779 & 0.160 & 49.167 \\
9.641 & 0.150 & 52.539 & 9.565 & 0.140 & 56.288 \\
11.773 & 0.167 & 47.273 & 11.720 & 0.128 & 61.712 \\
13.567 & 0.182 & 43.452 & 13.489 & 0.174 & 45.446 \\
14.789 & 0.140 & 56.563 & 14.716 & 0.180 & 43.990 \\
16.609 & 0.180 & 44.090 & 16.540 & 0.182 & 43.601 \\
17.623 & 0.172 & 46.202 & 17.540 & 0.167 & 47.580 \\
19.703 & 0.199 & 40.053 & 19.109 & 0.177 & 44.991 \\
26.125 & 0.203 & 39.712 & 26.028 & 0.177 & 45.537 \\
29.505 & 0.210 & 38.670 & 29.431 & 0.160 & 50.745 \\
\hline average crystal size: & & $\mathbf{4 4 . 7 8 9}$ & & & $\mathbf{4 8 . 9 0 6}$ \\
\hline
\end{tabular}

$\tau$ : crystal size

\subsection{BET Surface Area}

Table (3), summarizes BET surface area $\left(\mathrm{m}^{2} / \mathrm{g}\right)$, pore volume $\left(\mathrm{cm}^{3} / \mathrm{g}\right)$, and mean pore diameter (nm) of all samples. With regard to $A C / C u B T C$ composite, it can be seen that the amount of BET surface area and the pore volume associated with $A C / C u B T C$ composite is lower than bare $C u B T C$ sample. It is worth mentioning that, there is an astonishing discrepancy between the data available in literature around the effect of incorporating different carbonaceous materials within different MOFs on textural properties of resulting composites. Table (4) qualitatively summarizes the effect of incorporating different carbonaceous materials within MOF structures on textural properties of final composite materials. We believe that, the resulting textural properties of composite materials, not only is owing to diverse preliminary textural properties of carbonaceous materials and dissimilar synthesis procedures, but also can be resulted from different properties of bare MOFs. 
Table3 Textural properties of as-synthesized samples

\begin{tabular}{cccc}
\hline Sample & BET $\left(\mathbf{m}^{2} / \mathbf{g}\right)$ & Pore Volume $\left(\mathbf{c m}^{3} / \mathbf{g}\right)$ & Mean Pore Diameter $(\mathbf{n m})$ \\
\hline $\boldsymbol{A C}$ & 1635 & 0.9043 & 2.2118 \\
$\boldsymbol{C u B T C}$ & 1689 & 0.7095 & 1.6805 \\
$\boldsymbol{A C - 0 5 0 / C u B T C}$ & 1630 & 0.6634 & 1.6284 \\
\hline
\end{tabular}

Table4 Effect of incorporating different carbonaceous materials within MOF structures on textural properties of composite materials

\begin{tabular}{cccc}
\hline Composite & Surface Area & Pore Volume & Reference \\
\hline AC@MIL-101 & increase & decrease & {$[25]$} \\
AC@MIL-101 & increase & increase & {$[14]$} \\
AC/MIL-101 $(\mathrm{Cr})$ & decrease & --- & {$[17]$} \\
AC/MIL 88B $(\mathrm{Fe})$ & increase & decrease & {$[26]$} \\
AC@MIL-101 $(\mathrm{Cr})$ & increase & increase & {$[27]$} \\
CNT@CuBTC & decrease & increase & {$[7]$} \\
CNT@MOF-5 & increase & increase & {$[8]$} \\
GrO@CuBTC & increase & increase & {$[28]$} \\
GrO@ZIF-8 & decrease & decrease & {$[18]$} \\
AC@CuBTC & decrease & decrease & this work \\
\hline
\end{tabular}

\subsection{FESEM Analysis}

Detailed morphology and microstructure of prepared samples were explored by means of FESEM analysis. Figures (3a-b) and figures (3c-d) are associated with $C u B T C$ and $A C / C u B T C$ samples, respectively. As can be seen, both $C u B T C$ and $A C / C u B T C$ samples comprising of octahedral structures similar to CuBTC images reported before [23, 29, 30]. Besides, it is clear that the crystal size of bare $C U B T C$ sample is lower than traditional crystal size of CuBTC synthesized at temperatures above $100{ }^{\circ} \mathrm{C}$ (i.e. $\left.1 \mu \mathrm{m}\right)$ [31]. 

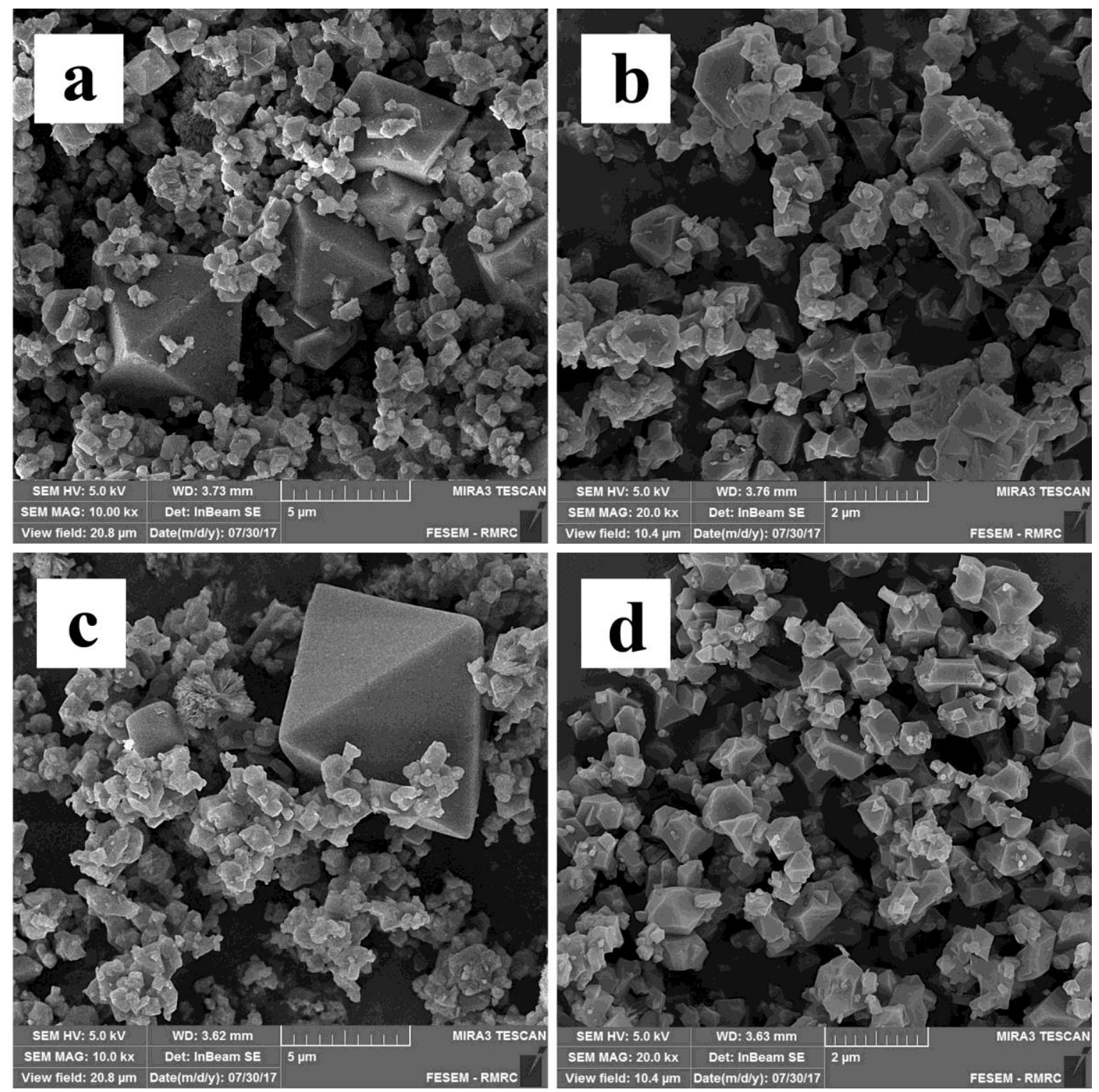

Fig3 FESEM images of a-b) $C u B T C$ and c-d) $A C / C u B T C$ samples

\subsection{Effect of AC Incorporation on $\mathrm{CO}_{2} / \mathrm{H}_{2}$ Separation behavior}

For separation of gaseous mixtures, there are two main phenomena influencing the separation behavior of a porous solid; molecular sieving effect and preferential adsorption. Zhao et al. [32], reported that for gas separation purposes, MOF structures possess pores with larger than $0.7 \mathrm{~nm}$ (such as CuBTC), show the best separation performance based on preferential adsorption. In 
addition, Yan et al. [33] demonstrated that at low pressures $(<1.2$ bar $)$ and low temperatures, chemical characteristics of pore surfaces would mainly dictate the adsorptive properties of MOFs.

Figure (4) represents the separation behavior of bare $C u B T C$ sample as well as $A C / C u B T C$ composites for $\mathrm{CO}_{2} / \mathrm{H}_{2}$ separation at atmospheric pressure and $273 \mathrm{~K}$. In the case of $C u B T C$ sample, observed high $\mathrm{CO}_{2} / \mathrm{H}_{2}$ selectivity (i.e. $\approx 15$ ) might be resulted from the presence of open $\mathrm{Cu}^{2+}$ metal sites with high affinity to $\mathrm{CO}_{2}$ molecules as reported by Liu et al. [34]. However, by increasing the amount of $\mathrm{AC}$ in $A C / C u B T C$ composite, $\mathrm{CO}_{2} / \mathrm{H}_{2}$ separation factor experienced a decreasing-increasing trend. To describe this observation, one should bear in mind that the separation factor is relied on competitive adsorption of each component within the gas mixture. In other words, since textural properties of as-synthesized $A C / C u B T C$ composites are different, the affinity of each sample to adsorb $\mathrm{CO}_{2}$ and/or $\mathrm{H}_{2}$ molecules would be different too. In the case of decreasing the $\mathrm{CO}_{2} / \mathrm{H}_{2}$ selectivity by adding $0.005 \mathrm{~g}$ of $\mathrm{AC}$, it should be mentioned that this finding is in good agreement with our previous research [19] where optimum amount of AC for achieving the highest hydrogen adsorption capacity of $A C / C u B T C$ composite was equal to $0.005 \mathrm{~g}$. In other words, higher affinity of $A C-005 / C u B T C$ sample to hydrogen adsorption can result in lower amounts of $\mathrm{CO}_{2} / \mathrm{H}_{2}$ selectivity. However, by increasing the AC content up to $0.05 \mathrm{~g}$, decreased affinity to adsorb hydrogen hand in hand with increasing the capacity for $\mathrm{CO}_{2}$ adsorption owing to the presence of more nitro and carbonyl functional groups on the surface of $A C / C u B T C$ composite, resulted in increasing the $\mathrm{CO}_{2} / \mathrm{H}_{2}$ selectivity up to around 32 which is almost double the separation factor of bare CUBTC sample (i.e. $\approx 15)$. It is worth mentioning that, although the presence of higher amounts of $\mathrm{AC}$ in synthesis solution can cause occupation of more $\mathrm{Cu}^{2+}$ unsaturated sites by functionalized AC particles, however, grafting functional groups available on the surface of 
$\mathrm{AC}$ (i.e. nitro and carbonyl groups) can result in higher affinity of $A C / C u B T C$ composites to $\mathrm{CO}_{2}$ molecules and hence higher $\mathrm{CO}_{2} / \mathrm{H}_{2}$ selectivity [20].

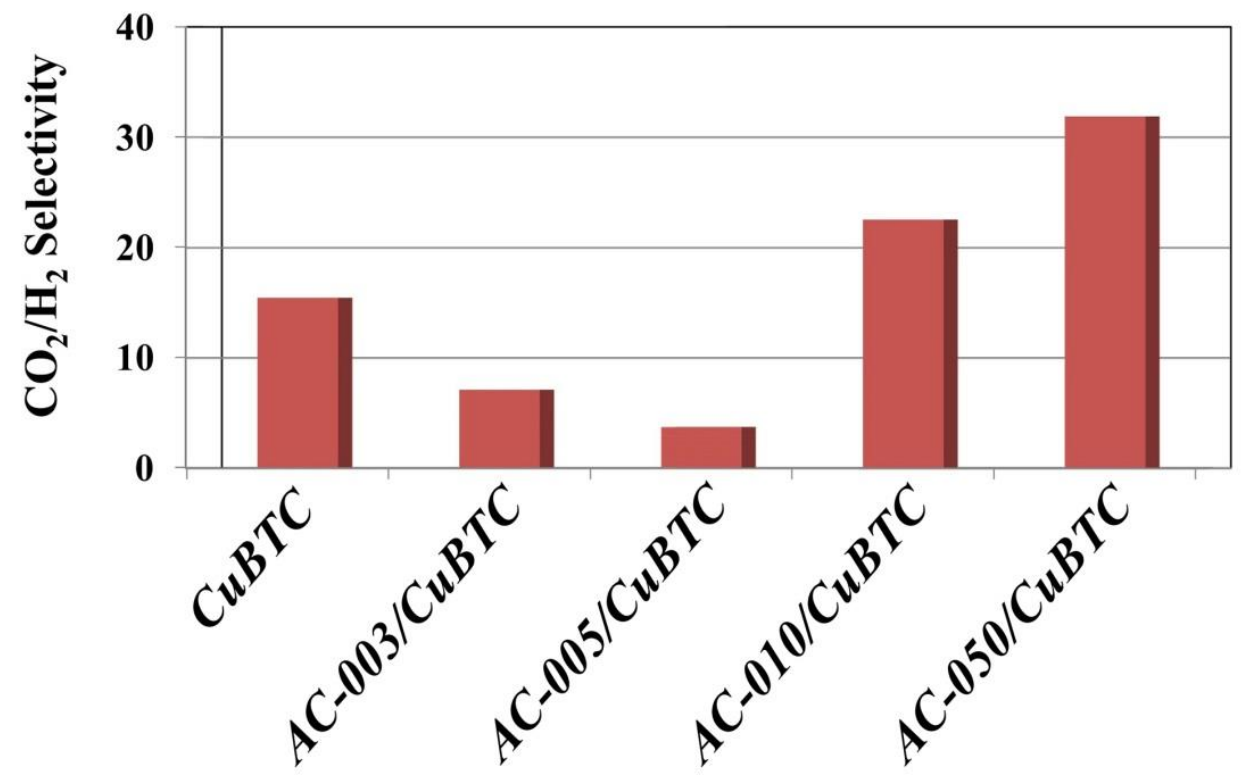

Fig4 $\mathrm{CO}_{2} / \mathrm{H}_{2}$ selectivity of bare $\mathrm{CuBTC}$ and as-synthesized $A C / C u B T C$ composites

To shed some light on mechanism of $\mathrm{CO}_{2} / \mathrm{H}_{2}$ separation using $A C / C u B T C$ composites, $\mathrm{CO}_{2}$ and $\mathrm{H}_{2}$ pure gas adsorption experiments were taken under consideration as well. Figures (5) and (6) represent the adsorption isotherms of pure $\mathrm{CO}_{2}$ and $\mathrm{H}_{2}$ at $298 \mathrm{~K}$, respectively. As can be seen, the capacity of functionalized $\mathrm{AC}$ for both $\mathrm{CO}_{2}$ and $\mathrm{H}_{2}$ experiments is lower than bare $C u B T C$ and composite samples. On the other hand, it can be concluded that using 0.003 $\mathrm{g}$ and $0.005 \mathrm{~g}$ of functionalized $\mathrm{AC}$ in $\mathrm{CuBTC}$ matrix, can enhance the $\mathrm{CO}_{2}$ and $\mathrm{H}_{2}$ capacity of composite materials up to around $9 \%$ and $37 \%$, respectively. However, by increasing the amount of $\mathrm{AC}$ incorporation, both $\mathrm{CO}_{2}$ and $\mathrm{H}_{2}$ adsorption capacity decreased (even lower than bare $C u B T C$ sample for $\mathrm{H}_{2}$ adsorption). This finding might be attributed to the presence of more functionalize AC with low adsorption capacity in the composite sample. However, it can be clearly seen that, in the case of $\mathrm{CO}_{2}$ adsorption, the capacity of samples with higher amounts of functionalized AC does not change significantly which confirms the crucial role 
of functional groups available on the surface of $\mathrm{AC}$ during $\mathrm{CO}_{2}$ adsorption on $A C / C u B T C$ samples. In the overall, higher $\mathrm{CO}_{2}$ adsorption capacity and lower $\mathrm{H}_{2}$ capacity of $A C / C u B T C$ sample with $0.050 \mathrm{~g}$ of $\mathrm{AC}$ resulted in higher $\mathrm{CO}_{2} / \mathrm{H}_{2}$ separation factor as revealed by volumetric-chromatography technique.

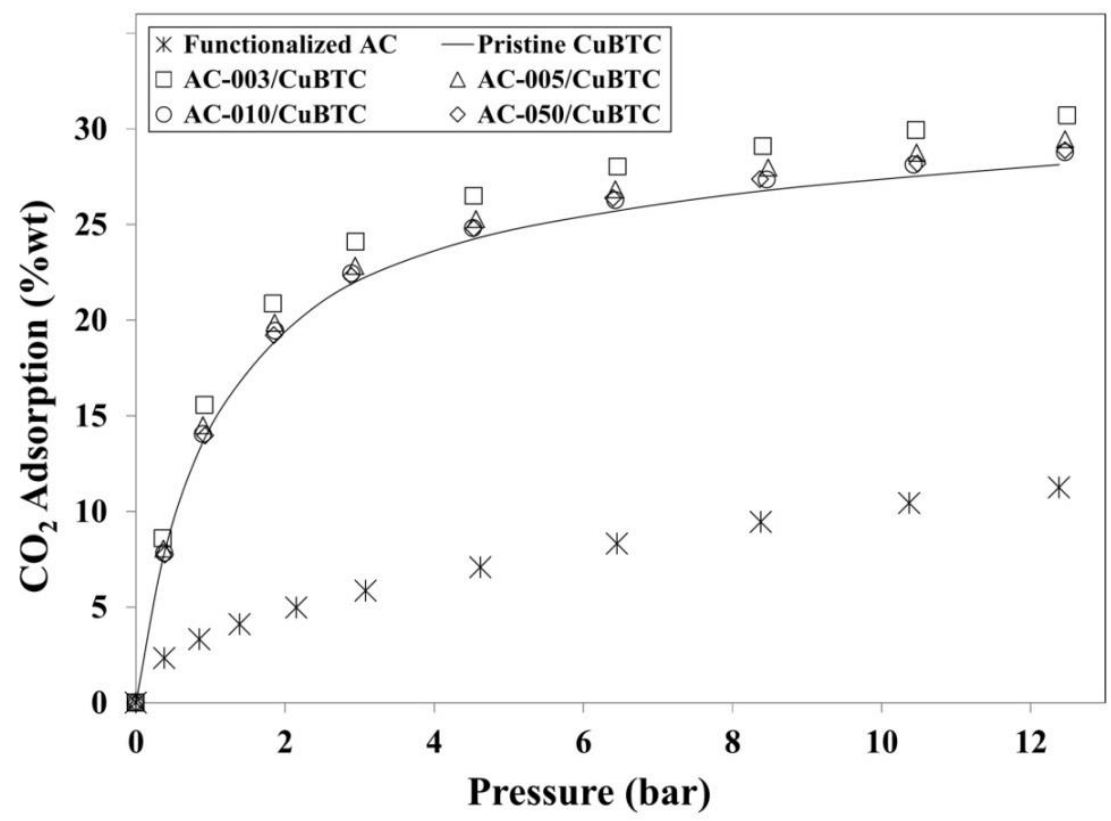

Fig5 $\mathrm{CO}_{2}$ capacities of as-synthesized samples

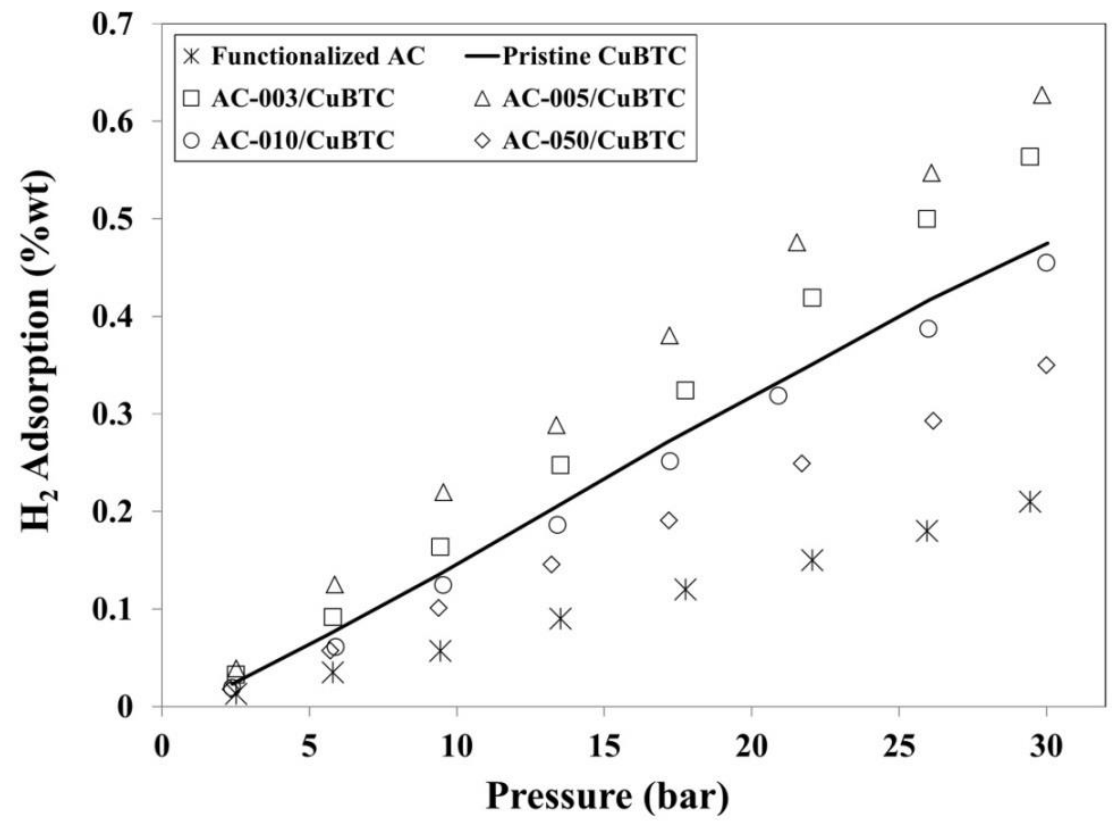

Fig6 $\mathrm{H}_{2}$ capacities of as-synthesized samples 


\section{Conclusion}

In this study, $A C / C u B T C$ composites were synthesized solvothermally and their performance was evaluated from viewpoint of $\mathrm{CO}_{2} / \mathrm{H}_{2}$ separation. The results showed that, while incorporation of AC within CuBTC structure does not significantly affect the crystalline structure of primary CuBTC, it can enhance the selectivity of $\mathrm{CO}_{2}$ over $\mathrm{H}_{2}$. By means of a volumetricchromatographic measurement, it was demonstrated that adding proper amount of AC within synthesis solution of CuBTC can result in higher separation factor for $\mathrm{CO}_{2} / \mathrm{H}_{2}$ mixture to reach values around 30 which is double the selectivity of pristine $C u B T C$ structure. This high affinity might be due to quadrupole moment of $\mathrm{CO}_{2}$, presence of $\mathrm{Cu}^{2+}$ unsaturated sites in MOF matrix, and grafting carbonyl and nitro functional groups available on the surface of AC incorporated. The results of the present study is promising because by incorporating low cost carbonaceous material into CuBTC structure, the separation factor can be substantially improved in comparison to both pure $\mathrm{AC}$ and $\mathrm{CuBTC}$ structures.

\section{Declarations}

\section{- Funding}

The authors did not receive support from any organization for the submitted work.

\section{- Conflicts of interest/Competing interests}

The authors have no conflicts of interest to declare that are relevant to the content of this article.

\section{- Availability of data and material}

All data generated or analyzed during this study are included in this published article.

\section{References}

1. Yang, Q. and C. Zhong, Molecular simulation of carbon dioxide/methane/hydrogen mixture adsorption in metal-organic frameworks. The Journal of Physical Chemistry B, 2006. 110(36): p. 17776-17783.

2. Sircar, S. and T. Golden, Purification of hydrogen by pressure swing adsorption. Separation Science and Technology, 2000. 35(5): p. 667-687. 
3. Hufton, J., S. Mayorga, and S. Sircar, Sorption-enhanced reaction process for hydrogen production. AIChE Journal, 1999. 45(2): p. 248-256.

4. Kuppler, R.J., et al., Potential applications of metal-organic frameworks. Coordination Chemistry Reviews, 2009. 253(23-24): p. 3042-3066.

5. Zhao, D., et al., Stabilization of metal- organic frameworks with high surface areas by the incorporation of mesocavities with microwindows. Journal of the American Chemical Society, 2009. 131(26): p. 9186-9188.

6. Xiang, Z., et al., CNT@Cu3(BTC)2 and metal-organic frameworks for separation of $\mathrm{CO} / \mathrm{CH} 4$ mixture. The Journal of Physical Chemistry C, 2011. 115(40): p. 19864-19871.

7. Xiang, Z., et al., Metal-organic frameworks with incorporated carbon nanotubes: improving carbon dioxide and methane storage capacities by lithium doping. Angewandte Chemie International Edition, 2011. 50(2): p. 491-494.

8. Yang, S.J., et al., Preparation and enhanced hydrostability and hydrogen storage capacity of CNT@MOF-5 hybrid composite. Chemistry of Materials, 2009. 21(9): p. 1893-1897.

9. Prasanth, K., et al., Enhanced hydrogen sorption in single walled carbon nanotube incorporated MIL-101 composite metal-organic framework. International journal of hydrogen energy, 2011. 36(13): p. 7594-7601.

10. Huang, A., et al., Synthesis of highly hydrophobic and permselective metal-organic framework Zn (BDC)(TED) 0.5 membranes for H2/CO2 separation. Journal of Membrane Science, 2014. 454: $\mathrm{p}$. 126-132.

11. Bandosz, T.J. and C. Petit, MOF/graphite oxide hybrid materials: exploring the new concept of adsorbents and catalysts. Adsorption, 2011. 17(1): p. 5-16.

12. Zhao, Y., et al., Aminated graphite oxides and their composites with copper-based metal-organic framework: in search for efficient media for CO 2 sequestration. RSC Advances, 2013. 3(25): p. 9932-9941.

13. Bajaj, H.C., et al., Activated carbon-metal organic framework composite materials with enhanced gas adsorption capacity and process for the preparation thereof. 2014, Google Patents.

14. Somayajulu Rallapalli, P., et al., Activated carbon@MIL-101(Cr): a potential metal-organic framework composite material for hydrogen storage. International journal of energy research, 2013. 37(7): p. 746-753.

15. Lee, S.-Y. and S.-J. Park, Effect of platinum doping of activated carbon on hydrogen storage behaviors of metal-organic frameworks-5. International journal of hydrogen energy, 2011. 36(14): p. 8381-8387.

16. Prabhakaran, P.K. and J. Deschamps, Doping activated carbon incorporated composite MIL-101 using lithium: impact on hydrogen uptake. Journal of Materials Chemistry A, 2015. 3(13): p. 7014-7021.

17. Mahmoodi, N.M., M. Taghizadeh, and A. Taghizadeh, Activated carbon/metal-organic framework composite as a bio-based novel green adsorbent: Preparation and mathematical pollutant removal modeling. Journal of Molecular Liquids, 2019. 277: p. 310-322.

18. McHugh, L.N., et al., Metal-Organic Framework-Activated Carbon Composite Materials for the Removal of Ammonia from Contaminated Airstreams. Angewandte Chemie International Edition, 2019. 58(34): p. 11747-11751.

19. Esfandiari, K., et al., Optimizing parameters affecting synthetize of CUBTC using response surface methodology and development of AC@ CUBTC composite for enhanced hydrogen uptake. International Journal of Hydrogen Energy, 2018.

20. Shafeeyan, M.S., et al., A review on surface modification of activated carbon for carbon dioxide adsorption. Journal of Analytical and Applied Pyrolysis, 2010. 89(2): p. 143-151. 
21. Keller, J.U. and R. Staudt, Volumetric-Gravimetric Measurements, in Gas Adsorption Equilibria, Experimental Methods and Adsorptive Isotherms. 2005, Springer. p. 181-235.

22. Hartmann, M., et al., Adsorptive separation of isobutene and isobutane on Cu3(BTC)2. Langmuir, 2008. 24(16): p. 8634-8642.

23. Pirzadeh, K., et al., Electrochemical synthesis, characterization and application of a microstructure $\mathrm{Cu} 3$ (BTC) 2 metal organic framework for $\mathrm{CO} 2$ and $\mathrm{CH} 4$ separation. Korean Journal of Chemical Engineering, 2018: p. 1-10.

24. Khoshhal, S., et al., Study of the temperature and solvent content effects on the structure of $\mathrm{Cu}-$ BTC metal organic framework for hydrogen storage. RSC Advances, 2015. 5(31): p. 24758-24768.

25. Prabhakaran, P.K., L. Catoire, and J. Deschamps, Aluminium doping composite metal-organic framework by alane nanoconfinement: Impact on the room temperature hydrogen uptake. Microporous and Mesoporous Materials, 2017. 243: p. 214-220.

26. Mahmoodi, N.M., et al., Activated carbon/metal-organic framework nanocomposite: Preparation and photocatalytic dye degradation mathematical modeling from wastewater by least squares support vector machine. Journal of environmental management, 2019. 233: p. 660672.

27. Hasanzadeh, M., A. Simchi, and H.S. Far, Nanoporous composites of activated carbon-metal organic frameworks for organic dye adsorption: Synthesis, adsorption mechanism and kinetics studies. Journal of Industrial and Engineering Chemistry, 2020. 81: p. 405-414.

28. Huang, W., et al., Preparation and adsorption performance of GrO@Cu-BTC for separation of CO2/CH4. Industrial \& Engineering Chemistry Research, 2014. 53(27): p. 11176-11184.

29. Lin, K.-S., et al., Synthesis and characterization of porous HKUST-1 metal organic frameworks for hydrogen storage. International journal of hydrogen energy, 2012. 37(18): p. 13865-13871.

30. Kim, J., H.-Y. Cho, and W.-S. Ahn, Synthesis and adsorption/catalytic properties of the metal organic framework CUBTC. Catalysis Surveys from Asia, 2012. 16(2): p. 106-119.

31. Schlichte, K., T. Kratzke, and S. Kaskel, Improved synthesis, thermal stability and catalytic properties of the metal-organic framework compound CU3(BTC)2. Microporous and Mesoporous Materials, 2004. 73(1-2): p. 81-88.

32. Zhao, Z., et al., Gas separation properties of metal organic framework (MOF-5) membranes. Industrial \& Engineering Chemistry Research, 2012. 52(3): p. 1102-1108.

33. Yan, X., et al., Extremely enhanced CO2 uptake by HKUST-1 metal-organic framework via a simple chemical treatment. Microporous and Mesoporous Materials, 2014. 183: p. 69-73.

34. Liu, Y., Z.U. Wang, and H.C. Zhou, Recent advances in carbon dioxide capture with metal-organic frameworks. Greenhouse Gases: Science and Technology, 2012. 2(4): p. 239-259. 
Figures

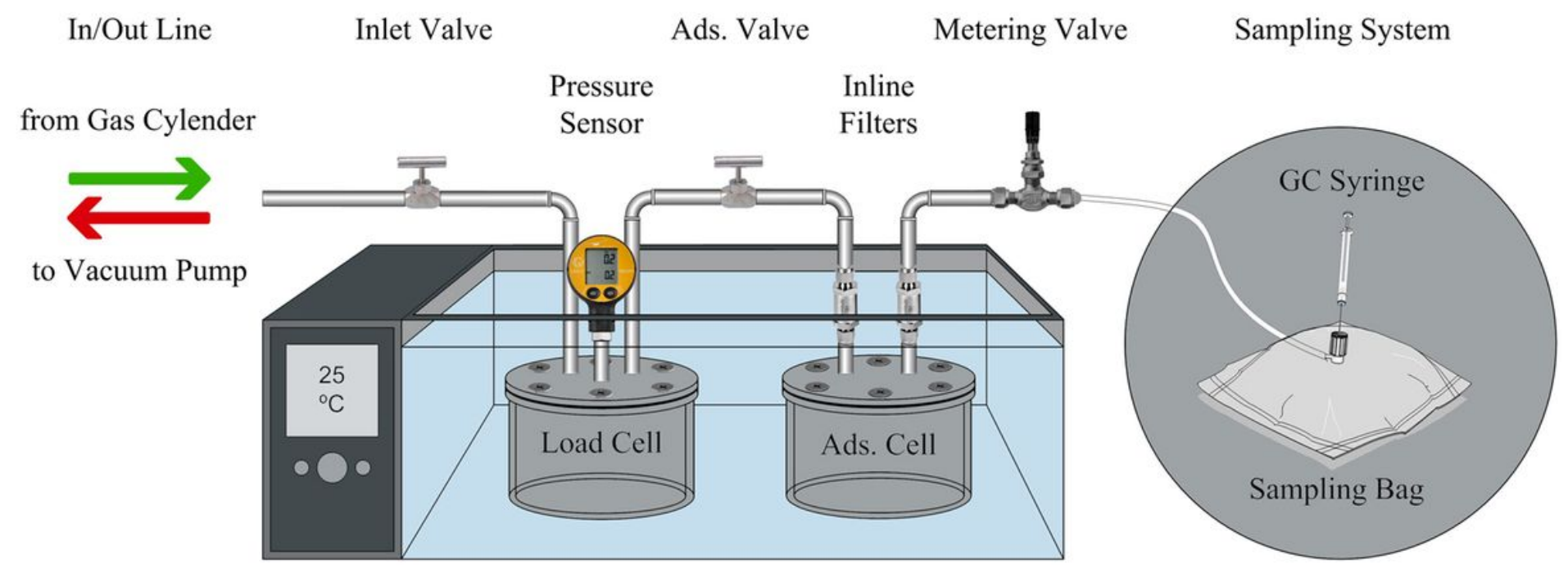

Figure 1

Schematic diagram of volumetric adsorption apparatus equipped to a sampling line

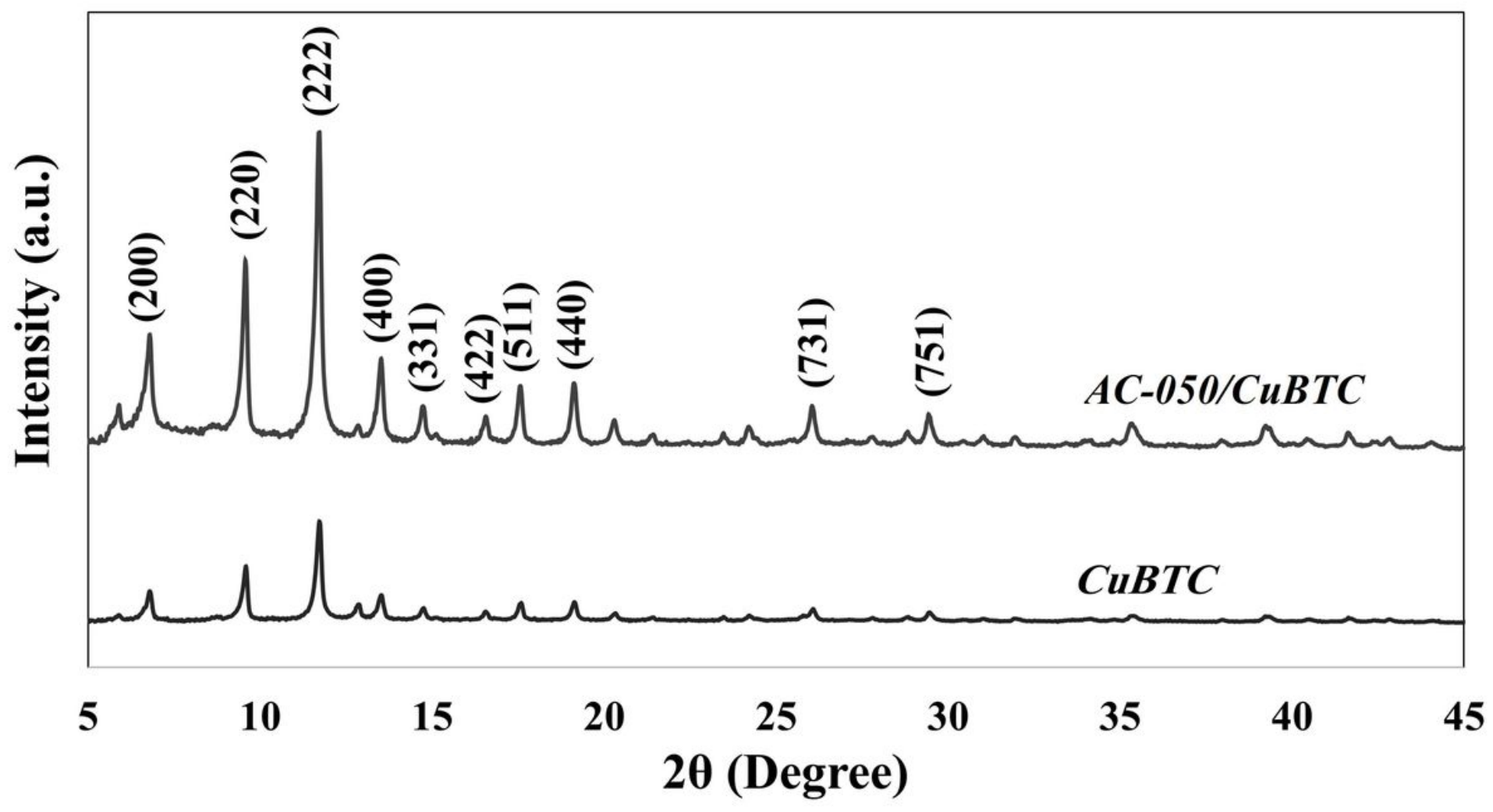

Figure 2

PXRD patterns of bare CuBTC and AC-050/CuBTC composite 

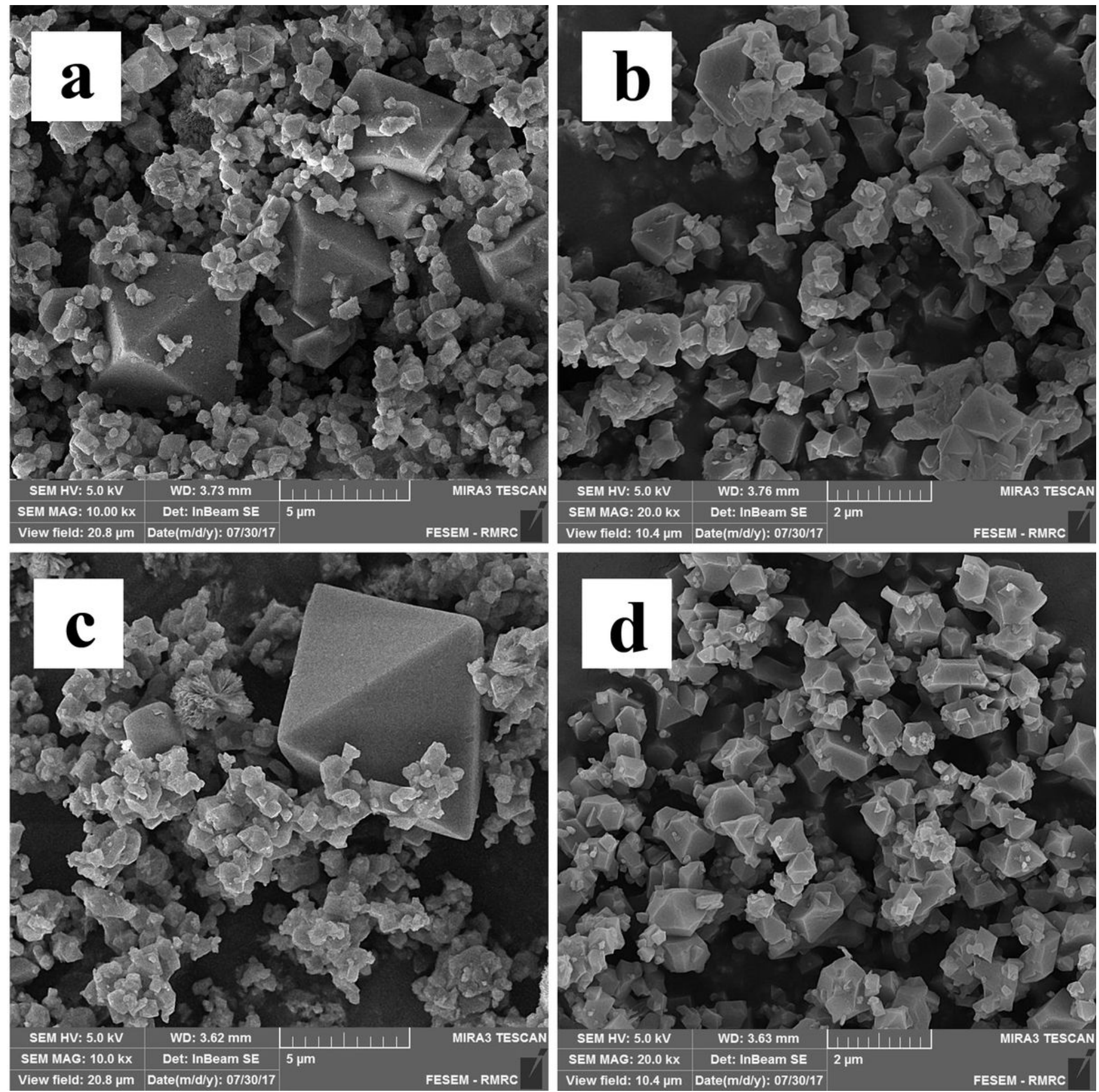

\section{Figure 3}

FESEM images of $a-b)$ CuBTC and c-d) AC/CuBTC samples 


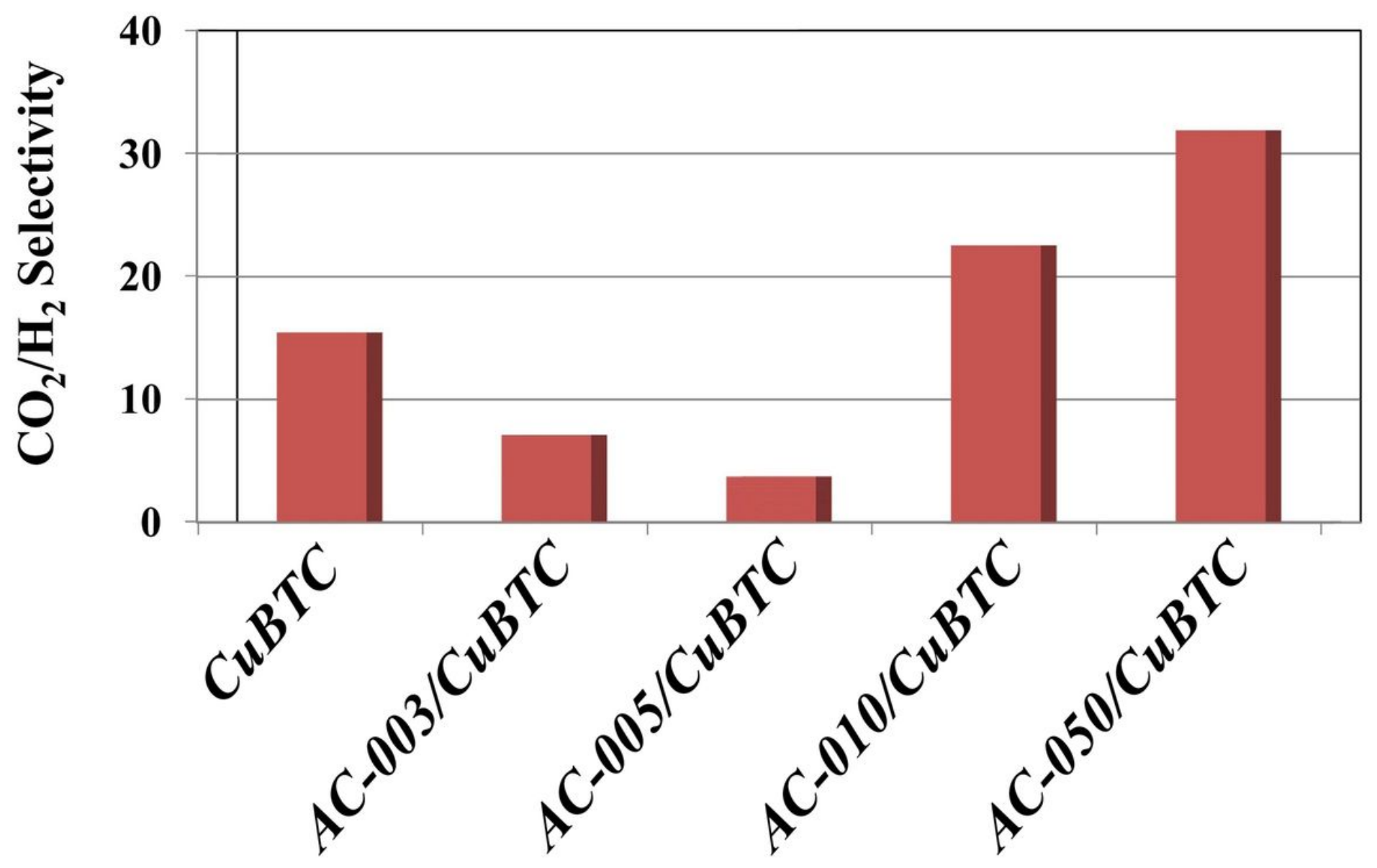

Figure 4

CO2/H2 selectivity of bare CuBTC and as-synthesized AC/CuBTC composites 


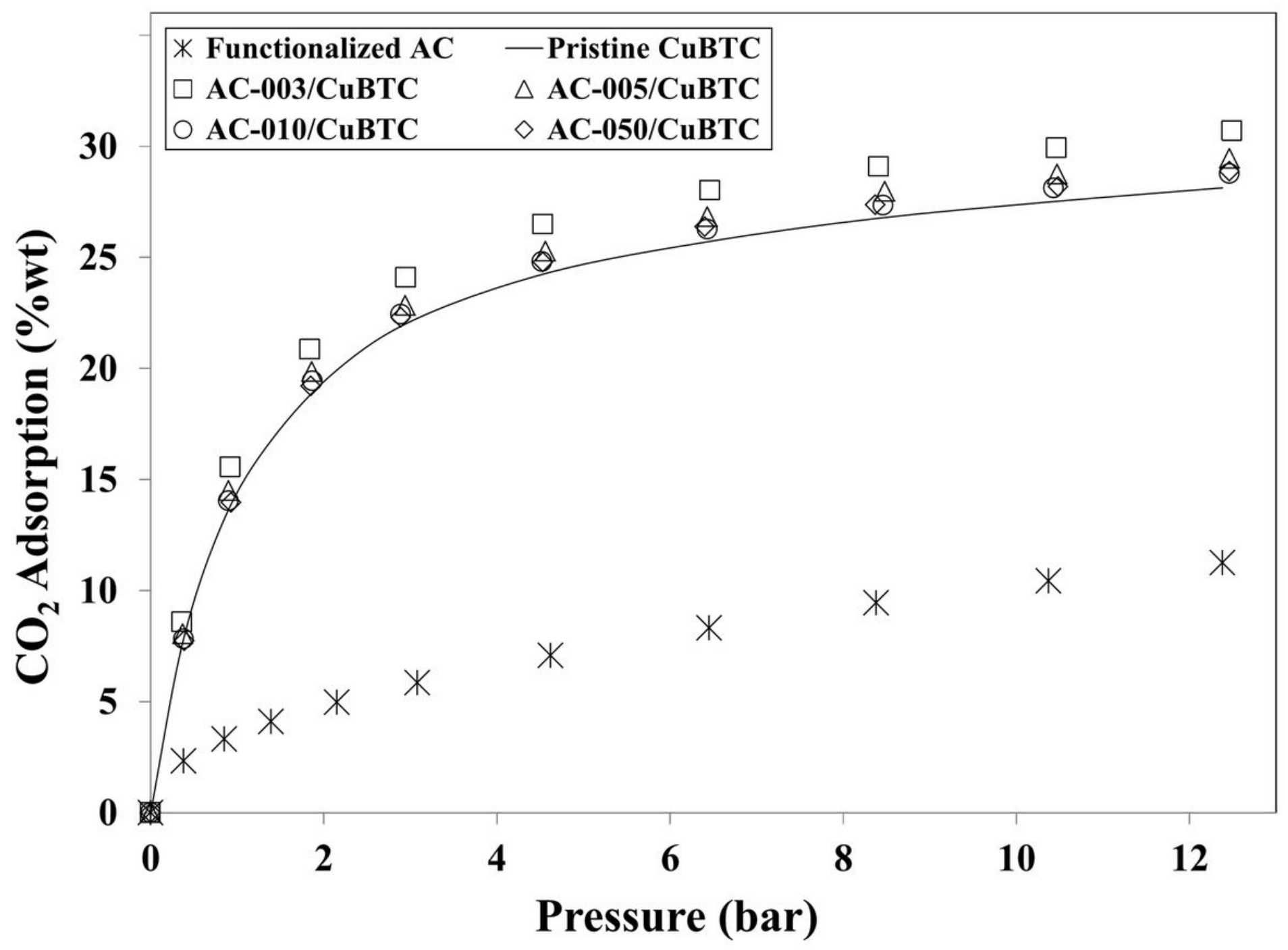

Figure 5

CO2 capacities of as-synthesized samples 


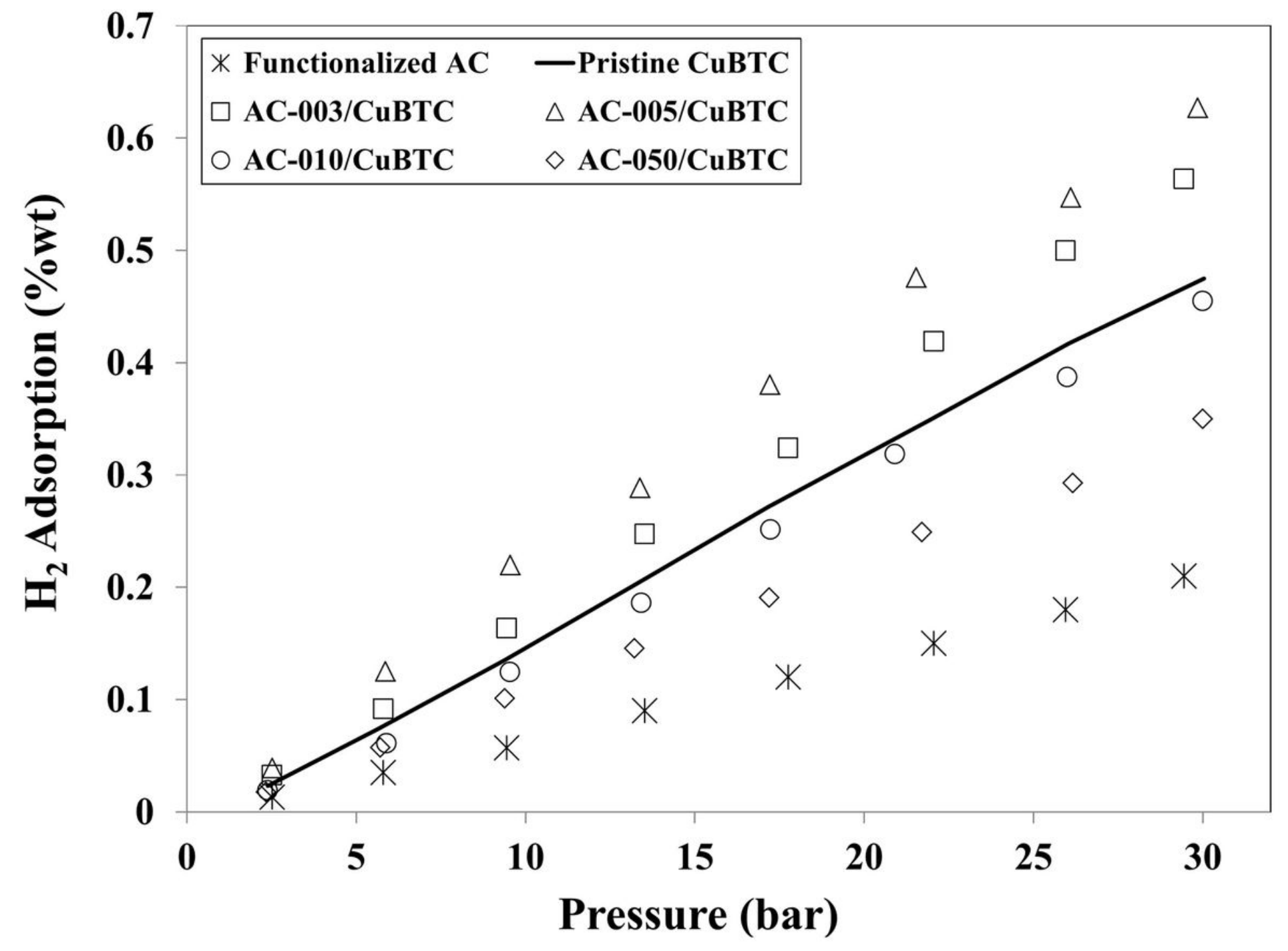

Figure 6

$\mathrm{H} 2$ capacities of as-synthesized samples 